\title{
Do CTA measurements of annular diameter, perimeter and area result in different TAVI prosthesis sizes?
}

\author{
Barbora Horehledova ${ }^{1,2}\left(\right.$ D Casper Mihl ${ }^{1,2} \cdot$ Babs M. F. Hendriks $^{1,2} \cdot$ Nienke G. Eijsvoogel $^{1,2} \cdot$ Jindrich Vainer $^{3}$. \\ Leo F. Veenstra ${ }^{3}$ Joachim E. Wildberger ${ }^{1,2} \cdot$ Marco Das $^{1,2,4}$
}

Received: 14 February 2018 / Accepted: 6 June 2018 / Published online: 16 June 2018

(C) The Author(s) 2018

\begin{abstract}
Incorrect prosthesis size has direct impact on patient outcome after transcatheter aortic valve implantation (TAVI) procedure. Currently, annular diameter, area or perimeter may be used for prosthesis size selection. The aim was to evaluate whether the use different annular dimensions would result in the selection of different prosthesis sizes, when assessed in the same TAVI-candidate during the same phase of a cardiac cycle. Fifty consecutive TAVI-candidates underwent retrospectively ECG-gated computed tomography angiography (CTA). Aortic root dimensions were assessed in the $20 \%$ phase of the R-R interval. Annular short diameter, perimeter and area were used to select the prosthesis size, based on the industry recommendations for a self-expandable (Medtronic CoreValve; MCV) and balloon-expandable (Edwards Sapien XT Valve; ESV) valve. Complete agreement on selected prosthesis size amongst all three annular dimensions was observed in 62\% (31/50; ESV) and 30\% (15/50; MCV). Short aortic annulus measurement resulted in a smaller prosthesis size in 20\% (10/50; ESV) and in $60 \%$ of cases (30/50; MCV) compared to the size suggested by both annular perimeter and area. In 18\% (9/50; ESV) and $10 \%$ of cases $(5 / 50 ; \mathrm{MCV})$ a larger prosthesis would have been selected based on annular perimeter compared to annular diameter and area. Prosthesis size derived from area was always in agreement with at least one other parameter in all cases. Aortic annulus area appears to be the most robust parameter for TAVI-prosthesis size selection, regardless of the specific prosthesis size. Short aortic annulus diameter may underestimate the prosthesis size, while use of annular perimeter may lead to size overestimation in some cases.
\end{abstract}

Keywords Aortic valve stenosis · Heart valve prosthesis implantation · Transcatheter aortic valve replacement . Multidetector computed tomography

\section{Abbreviations}

CM Contrast media

CTA Computed tomographic angiography

$\mathrm{D}_{\mathrm{A}} \quad$ Effective diameter derived from aortic annulus area

Barbora Horehledova

barbora.horehledova@mumc.nl

1 Department of Radiology and Nuclear Medicine, Maastricht University Medical Center, P.O. Box 5800, 6202 AZ Maastricht, The Netherlands

2 CARIM School for Cardiovascular Diseases, Maastricht University Medical Center, Maastricht, The Netherlands

3 Department of Cardiology, Maastricht University Medical Center, Maastricht, The Netherlands

4 Department of Diagnostic and Interventional Radiology, Helios Kliniken Duisburg GmbH, Duisburg, Germany
$\mathrm{D}_{\mathrm{P}} \quad$ Effective diameter derived from aortic annulus perimeter

ESV Edwards Sapien valve

IDR Iodine delivery rate

IR Iterative reconstruction

LVOT Left ventricular outflow tract

MCV Medtronic CoreValve

METC Medical ethical research committee

MDCT Multidetector row computed tomography

MPR Multiplanar reformations

PLAX Parasternal long axis view

ROI Region of interest

SCCT Society of cardiovascular computed tomography

SD Standard deviation

SPSS Statistical package for social sciences

TAVI Transcatheter aortic valve implantation 


\section{Introduction}

Transcatheter aortic valve implantation (TAVI) is a minimally invasive and alternative treatment of severe symptomatic aortic stenosis, originally only indicated in patients with high surgical risk, who are not suitable for an openheart surgical aortic valve replacement [1-3]. However, with the publication of new 2017 ESC/EACTS Guidelines for the management of valvular heart disease, the indications for TAVI have expanded, because there is new evidence for TAVI also in the intermediate risk population [4]. Imaging plays a key role in pre-procedural planning, reliable selection of TAVI prosthesis and choice of suitable valve size [2, $3,5,6]$. Precise pre-procedural imaging is therefore crucial to assure optimal patient outcome $[2,3,5]$.

Early industry recommendations for transcatheter aortic valve size selection have been based on the annular diameter, which was assessed as the left ventricular outflow tract (LVOT) diameter in echocardiography [6]. Multidetector row computed tomography (MDCT), with the possibility of three-dimensional visualization, has proven to be superior in aortic root assessment and prediction of patient outcome [5, 7]. MDCT has therefore become the method of choice in TAVI planning [5, 8]. Implementation of routine threedimensional evaluation of the aortic annulus allowed the manufacturers to extend the prosthesis sizing guidelines to three annular dimensions, namely annular diameter, area and perimeter $[6,9]$.

However, no further directions for their individual application or explanation of their associated relationship have been propounded in the industry guidelines. This has led to a flexible interpretation of industry guidelines. Annular area and perimeter have served either directly for prosthesis size selection or for further calculation of effective diameters. Therefore, up to five annular diameters (short, long, mean, area and perimeter derived effective diameters) have been frequently referred to in the literature as optional dimensions for TAVI planning [10-13]. Industry recommended diameter sizing thresholds have been applied to all five above-mentioned diameters without any adaptation, even though the systematic difference, described as increasing diameter length respectively from the short to effective and long diameter, has been regularly reported, possibly suggesting various prosthesis sizes $[10,11,13]$.

To restrict from modification of industry recommendations in this study, we derive the TAVI prosthesis size directly from the measurement of annular area or perimeter, rather than from calculated effective diameters. For annular diameter, we evaluate the MDCT assessed short annular diameter, because it resembles the LVOT diameter assessed on the parasternal long axis view (PLAX) in echocardiography $[6,10]$ and therefore corresponds the best with the diameter sizing thresholds in industry guidelines.

To the best of our knowledge this is the first study to evaluate, whether the three aortic annulus dimensions stated in the industry guidelines can indeed be used interchangeably for TAVI planning. To this aim, we have assessed the aortic annulus short diameter, area and perimeter in the same phase of the cardiac cycle in fifty consecutive TAVI-candidates undergoing MDCT and recorded the suggested prosthesis size for each annular measurement.

\section{Materials and methods}

\section{Patient population}

Fifty consecutive TAVI candidates with severe and symptomatic aortic valve stenosis, who underwent pre-TAVI MDCT assessment of the aortic root, were retrospectively included in this study. Ethical approval and a waiver of informed consent were given by the local medical ethical research committee (METC reference number: 2017-0265).

\section{MDCT scan and contrast media protocol}

A uniform dedicated scan protocol on a 2nd generation dual source MDCT (SOMATOM Definition Flash, Siemens, Forchheim, Germany), consisting of a low-pitch retrospective ECG-gated helical scan of the aortic root, followed by a high-pitch non ECG-triggered computed tomographic angiography (CTA) of the whole aorta, was used. The acquisition protocol is summarized in Table 1 . The total of $120 \mathrm{ml}$ of pre-warmed, monomeric, non-ionic, low osmolar iodinated contrast media (CM; Iopromide, Ultravist 300, Bayer, Berlin, Germany) was injected through an $18 \mathrm{G}$ needle in the antecubital vein following previously published tri-phasic CM injection protocol [8].

\section{Image reconstruction}

Images were reconstructed with dedicated post processing software (SyngoVia ${ }^{\mathrm{TM}}$, version VB10A Siemens, Forchheim, Germany) with $0.75 \mathrm{~mm}$ slice thickness and $0.7 \mathrm{~mm}$ increment using a raw-data based iterative reconstruction (IR) algorithm (SAFIRE, Siemens Healthcare, Forchheim, Germany) with B26f kernel, strength 3. Images from the low-pitch retrospectively ECG-gated scan were reconstructed at 10 time points throughout the R-R interval at $10 \%$ increments $(0-100 \%$ phase). 
Table 1 Scan and contrast media protocols

\begin{tabular}{|c|c|c|}
\hline \multirow{2}{*}{$\begin{array}{l}\text { Scan type } \\
\text { Scan direction }\end{array}$} & Retrospective ECG-gated & \multirow{2}{*}{$\begin{array}{l}\text { Non } \\
\text { ECG- } \\
\text { triggered }\end{array}$} \\
\hline & Cranio-caudal & \\
\hline Tube voltage $(\mathrm{kv})$ & \multicolumn{2}{|c|}{100} \\
\hline Quality ref. tube current (mAs) & 320 & 150 \\
\hline Dose modulation & \multicolumn{2}{|c|}{ CARE Dose4D } \\
\hline Rotation time (s) & \multicolumn{2}{|c|}{0.28} \\
\hline Pitch & 0.17 & 3 \\
\hline Slice collimation & \multicolumn{2}{|c|}{$2 \times 2 \times 64 \times 0.6$} \\
\hline Slice width $(\mathrm{mm})$ & $0.75 / 0.7$ & $1.5 / 1.0$ \\
\hline Reconstruction kernel & B26f & I30f \\
\hline Contrast media & \multicolumn{2}{|l|}{ Iopromide 300 (Ultravist) } \\
\hline Test bolus & \multicolumn{2}{|c|}{$20 \mathrm{ml} \mathrm{CM} \times 7.2 \mathrm{ml} / \mathrm{s}$ followed by $15 \mathrm{ml} \mathrm{NaCl} \times 7.2 \mathrm{ml} / \mathrm{s}$} \\
\hline Main bolus & \multicolumn{2}{|c|}{$\begin{array}{l}75 \mathrm{ml} \mathrm{CM} \times 7.2 \mathrm{ml} / \mathrm{s}(100 \%) \\
50 \mathrm{ml} \mathrm{CM} / \mathrm{NaCl} \times 7.2 \mathrm{ml} / \mathrm{s}(50 / 50 \%) \\
25 \mathrm{ml} \mathrm{NaCl} \times 7.2 \mathrm{ml} / \mathrm{s}\end{array}$} \\
\hline Iodine delivery rate $(\mathrm{gI} / \mathrm{s})$ & \multicolumn{2}{|l|}{2.16} \\
\hline Total iodine load $(\mathrm{gI})$ & \multicolumn{2}{|l|}{36} \\
\hline
\end{tabular}

$C M$ contrast medium, ECG electrocardiogram, $g I$ grams of iodine, $k V$ kilovolt, $m A s$ milliamper-second, $m G y$ milligray, $\mathrm{ml}$ milliliter, $\mathrm{mm}$ millimeter, $\mathrm{NaCl}$ saline, $s$ second

\section{Assessment of aortic annulus dimensions}

The aortic annulus dimensions were assessed using multiplanar reformations (MPR) view on the oblique transversal plane, positioned at the level of the aortic annulus in such a way that the most basal portions of the aortic leaflets were equally distributed in the transversal plane view, in accordance with the expert consensus guidelines of the Society of Cardiovascular Computed Tomography (SCCT) [6]. This position allowed measurements of the short and long annular diameter as well as of the perimeter and area of the aortic annulus. To perform the measurements the $20 \%$ phase of the $\mathrm{R}-\mathrm{R}$ interval (endsystole) was selected on the retrospectively ECG-gated low-pitch helical scans, according to our institutional standard [8].

The mean diameter was defined as an average of short and long annular diameter. The effective diameters derived from area $\left(D_{A}\right)$ and perimeter $\left(D_{P}\right)$ were calculated with commonly used and previously published formulas (Table 2) [8]. Annular eccentricity was quantified with the following equation:

Table 2 Formulas used for calculation of effective diameters and MCV sizing thresholds for annular area and diameter
Annular eccentricity $=\sqrt{\left[1-\left(\frac{\text { short annular diameter }}{\text { long annular diameter }}\right)^{2}\right]}$

An eccentricity greater than 0 describes an elliptical aortic annulus, while an eccentricity of 0 describes a perfect circle [2].

\section{Transcatheter aortic valve prosthesis sizing}

Transcatheter aortic valve sizes were theoretically selected based on the thresholds for the various dimensions of the aortic annulus as stated in the industry recommendations for balloon expandable (Edwards SapienValve XT; [ESV]; Edwards Lifesciences Corp, Irvine, USA) and self-expandable valve (Medtronic CoreValve; [MCV]; Medtronic, Minneapolis, USA) [6, 14, 15]. Recommendations are summarized in Table 3. The agreement between suggested valve sizes derived from individual annular dimensions was analyzed.

\begin{tabular}{lll}
\hline & $\begin{array}{l}\text { Formulas used for calculation of effective } \\
\text { diameters }\end{array}$ & $\begin{array}{l}\text { Formulas used for calculation of } \\
\text { MCV sizing thresholds }\end{array}$ \\
\hline Cross sectional area & $\mathrm{D}_{\mathrm{A}}=2 \times \sqrt{\left(\frac{\text { cross-sectional area }}{\pi}\right)}$ & Cross-sectional area $=\frac{\pi \times \text { diameter }^{2}}{4}$ \\
Perimeter & $\mathrm{D}_{\mathrm{P}}=\frac{\text { perimeter }}{\pi}$ & Perimeter $=\pi \times$ diameter \\
\hline
\end{tabular}

$D_{A}$ effective diameter derived from annular area, $D_{P}$ effective diameter derived from annular perimeter 
Table 3 Industry recomendations for transcatheter aortic valve prosthesis sizing

\begin{tabular}{|c|c|c|c|c|}
\hline \multicolumn{5}{|l|}{ Edwards Sapien XT } \\
\hline Valve size (= device diameter) & $20 \mathrm{~mm}$ & $23 \mathrm{~mm}$ & $26 \mathrm{~mm}$ & $29 \mathrm{~mm}$ \\
\hline \multicolumn{5}{|c|}{ Suitable annular dimensions } \\
\hline Aortic annulus diameter $[\mathrm{mm}]$ & $17-19$ & $18-22$ & $21-25$ & $24-27$ \\
\hline Aortic annulus area $\left(\mathrm{mm}^{2}\right)$ & $280-320$ & $310-410$ & $410-520$ & $520-650$ \\
\hline Aortic annulus perimeter (mm) & $54-68$ & $62-72$ & $72-81$ & $81-90$ \\
\hline \multicolumn{5}{|l|}{ Medtronic CoreValve } \\
\hline \multirow[t]{2}{*}{ Valve size (= device diameter) } & $23 \mathrm{~mm}$ & $26 \mathrm{~mm}$ & $29 \mathrm{~mm}$ & $31 \mathrm{~mm}$ \\
\hline & \multicolumn{4}{|c|}{ Suitable annular dimensions } \\
\hline Aortic annulus diameter (mm) & $18-20$ & $20-23$ & $23-27$ & $26-29$ \\
\hline Aortic annulus area $\left(\mathrm{mm}^{2}\right)$ & 254-314 & $314-416$ & $416-572$ & $572-661$ \\
\hline Aortic annulus perimeter (mm) & $56-63$ & $63-72$ & $72-85$ & $82-91$ \\
\hline
\end{tabular}

$\mathrm{mm}$ millimeter, $\mathrm{mm}^{2}$ square millimeter

\section{Statistics}

Statistical analysis was conducted using Statistical Package for Social Sciences version 23.0 (SPSS Inc., Chicago, IL, USA). Continuous variables are expressed using descriptive statistics (mean value \pm standard deviation $[\mathrm{SD}]$ ). Categorical variables are expressed as frequencies and percentages. The mean values of aortic annulus dimensions were determined using a one-sample $t$ test. Mean differences between aortic annulus diameters were calculated with Bland-Altman method with 95\% limits of agreement (LOA; mean difference $\pm 1.96 \times \mathrm{SD})$. Agreement between suggested prosthesis sizes is expressed as the percentage of patients in which the same valve size would be selected based on two or three different annular measurements. All p-values are based on a 2 -sided $\alpha$ of $5 \%$, and a $p$-value $<0.05$ was considered statistically significant.

\section{Results}

\section{Baseline characteristics}

The baseline characteristics are summarized in Table 4. The study population consisted of 26 female and 24 male patients with an average age of $81 \pm 5$ years.

\section{Aortic annulus dimensions}

The mean aortic annulus measurement was $22.3 \pm 1.7 \mathrm{~mm}$, $472.5 \pm 63.8 \mathrm{~mm}^{2}$ and $79.3 \pm 5.9 \mathrm{~mm}$ for short aortic annulus diameter, area and perimeter, respectively. The mean eccentricity of the aortic annulus was $0.58 \pm 0.1$ (minimum: 0.36 ; maximum: 0.73).
Table 4 Baseline characteristics, pre- and post-procedural aortic valve characteristics on echocardiography

\begin{tabular}{|c|c|c|}
\hline & Mean \pm SD & Range \\
\hline Age (years) & $81 \pm 5$ & $69-88$ \\
\hline Height $(\mathrm{cm})$ & $167 \pm 10$ & $144-198$ \\
\hline Weight $(\mathrm{kg})$ & $74 \pm 12$ & $52-109$ \\
\hline \multicolumn{3}{|l|}{ Pre-procedural assessment } \\
\hline Ejection fraction (\%) & $55 \pm 12$ & $24-73$ \\
\hline Mean gradient (mmHg) & $42 \pm 17$ & $11-77$ \\
\hline $\begin{array}{l}\text { Maximum gradient } \\
(\mathrm{mmHg})\end{array}$ & $69 \pm 25$ & $21-120$ \\
\hline $\operatorname{AVA}\left(\mathrm{cm}^{2}\right)$ & $0.82 \pm 0.2$ & $0.4-1.5$ \\
\hline $\begin{array}{l}\text { Post-procedural assessment } \\
\text { (45) }\end{array}$ & $5 \pm 3$ days after intervention & \\
\hline Mean gradient (mmHg) & $10 \pm 4$ & $4-22$ \\
\hline $\begin{array}{l}\text { Maximum gradient } \\
(\mathrm{mmHg})\end{array}$ & $20 \pm 8$ & $9-45$ \\
\hline Aortic incompetence (AI) & $\begin{array}{l}\text { 1) Non/trace AI : } 20(44 \%) \\
\text { 2) Mild AI : } 21(47 \%) \\
\text { 3) Moderate AI: } 4(9 \%)\end{array}$ & \\
\hline
\end{tabular}

$A I$ aortic incompetence, $\mathrm{cm}$ centimeter, $\mathrm{cm}^{2}$ square centimeter, $\mathrm{mmHg}$ millimeter of mercury, $\mathrm{kg}$ kilogram, $S D$ standard deviation, $\%$ percent

The summary of the mean annular diameter (short, long, mean, $D_{A}, D_{P}$ ) values and their mean difference from the LVOT in echocardiography are presented in Table 6 in Appendix 1.

\section{Transcatheter aortic valve prosthesis sizing}

Complete agreement on selected prosthesis size by all three annular dimensions was observed in 31 (62\%; ESV) and 15 cases $(30 \%$; MCV). Short aortic annulus measurement 
resulted in a suggestion of one size smaller prosthesis size in 10 (20\%; ESV) and in 30 cases $(60 \%$; MCV), compared to the size suggested by both annular perimeter and area. In 9 (18\%; ESV) and 5 cases (10\%; MCV), one size larger prosthesis would be selected based on annular perimeter compared to both annular diameter and area. Prosthesis size derived from the aortic annulus area was in agreement with at least one other parameter in all cases. A summary of suggested valve sizes derived from short annular diameter, area and perimeter is presented in Table 5 and Fig. 1.

Table 5 Comparison of transcatheter aortic valve sizes derived from short annular diameter, area and perimeter

\begin{tabular}{lrr|rr}
\hline & ESV & \multicolumn{3}{c}{ MCV } \\
\hline Prosthesis size derived from & & & & \\
- Diameter $_{\mathrm{S}}=$ area = perimeter & 31 & $62 \%$ & 15 & $30 \%$ \\
- Diameter $_{\mathrm{S}}<$ (area = perimeter $)$ & 10 & $20 \%$ & 30 & $60 \%$ \\
- (Diameter $_{\mathrm{S}}=$ area $<$ perimeter & 9 & $18 \%$ & 5 & $10 \%$ \\
\hline
\end{tabular}

Diameter $_{S}$ short axis diameter, ESV Edwards Sapien Valve, $M C V$ Medtronic CoreValve
Table 7 and Fig. 4 in Appendix 2 presents the comparison of valve sizes derived from annular diameters (short, long, mean, $\mathrm{D}_{\mathrm{A}}, \mathrm{D}_{\mathrm{P}}$ ).

\section{Discussion}

This study clearly shows that annular diameter, area and perimeter dimensions are not interchangeable parameters in transcatheter aortic valve size selection, regardless of the prosthesis type. To the best of our knowledge, this is the first study that compares the differences in suggested prosthesis size between the three annular dimensions stated in the industry guidelines.

In this study, all three annular dimensions suggested the same prosthesis size in only $30-62 \%$ of cases. Based on these findings, $20-60 \%$ of the patients in this cohort could have been referred for a smaller valve size, perhaps leading to notable paravalvular regurgitation, device migration or embolization. In $10-18 \%$ of the study population, patients could be at risk of developing conduction disorders or even annular rupture, if referred for a larger than optimal valve
Fig. 1 Bar graph of aortic valve size distribution derived from aortic annulus short diameter, area and perimeter. $\mathrm{mm}$ millimeter
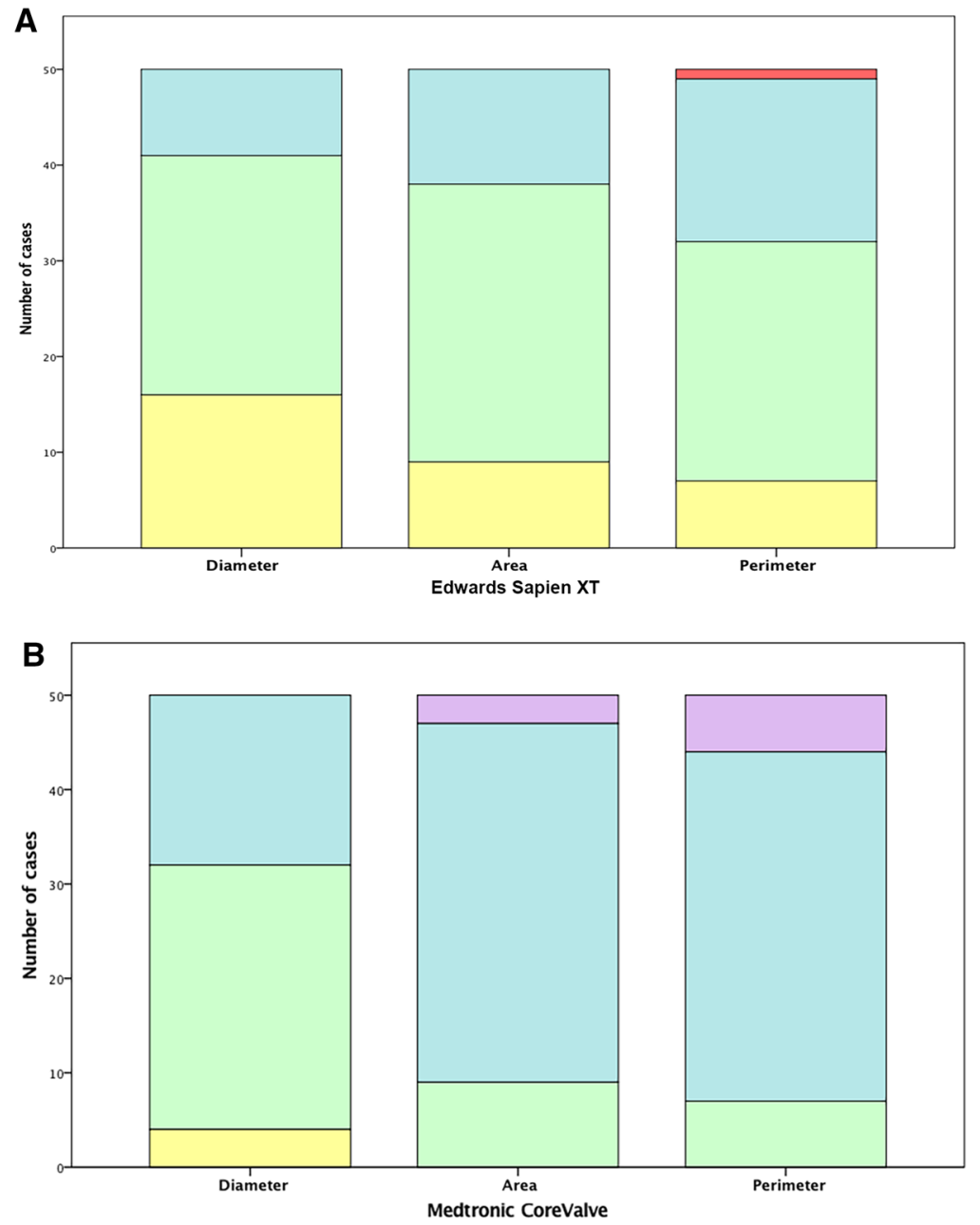

Valve size Not suitable for TAVI $29 \mathrm{~mm}$ $26 \mathrm{~mm}$
Valve size $\square 31 \mathrm{~mm}$ $\square 29 \mathrm{~mm}$ $\square 26 \mathrm{~mm}$ 
$[6,15]$. These results indicate that the matter of aortic valve sizing is considerably more complex than current guidelines would suggest.

The results of this study reveal that the transcatheter aortic valve sizing based on short annular diameter measurement has a tendency to underestimate the selected valve size, compared to a selection based on annular area and perimeter measurements. It is understandable that use of a one-dimensional parameter in prosthesis sizing might be prone to result in an unreliable estimation of prosthesis size, because it assumes circularity of the actually elliptical aortic annulus (Fig. 2). The use of three-dimensional annular dimension, and thus the use of MDCT should be therefore preferred over echocardiography in pre-procedural assessment [5-7]. Our results are in line with the findings of Schultz et al., who have reported the short diameter derived sizing to underestimate the prosthesis size in $50 \%$ of $\mathrm{MCV}$ cases when compared to direct surgical sizing [13]. This proportion of cases, potentially underestimating the prosthesis size, also corresponds with up to $67 \%$ incidence of paravalvular leak reported to follow the TAVI procedure [16, 17].

The results of this study also show that in some cases the measurement of annular perimeter has a tendency to result in larger valve size, compared to the consensual size derived from annular diameter and area. A combination of factors may be responsible for this overestimation. Manual assessment of the perimeter forms a rather polygonal than smooth annular silhouette [10]. Minimal irregularities and contour spikes can increase perimeter measurement leading to its overestimation and lower reproducibility, regardless of the post-processing software type [10, 15]. More importantly, the elliptical perimeter is proportionally amplified with increased eccentricity of the annular shape, in other words with greater difference between short and long diameter, even if the area of the ellipse remains the same (Fig. 3). This ellipse characteristic also complicates the use of effective diameters, which represent a diameter of an idealized circle with the same area or perimeter, regularly advised in literature for valve sizing [2, 11, 18]. When using effective diameters, it is important to consider the differences between devices, their characteristic in vivo shaping and their respective guideline set up. The MCV is a self-expandable valve, which adjusts to the elliptical annulus shape after deployment [3]. However, in the MCV guidelines the exact area and perimeter thresholds for each prosthesis size can be calculated with the dedicated formulas for a perfect circle from the annular diameter sizing thresholds. The general formulas for effective diameters are exactly reversed (see Table 2). From this, it follows that the absolute (100\%) agreement for suggested MCV size between area and $\mathrm{D}_{\mathrm{A}}$ is biased because they are mutually dependent, and the calculation of $\mathrm{D}_{\mathrm{A}}$ therefore does not bring any additional value for MCV sizing. On the other hand, the ESV guidelines do not possess such a predictable relationship between diameter and area (or perimeter), even though the balloon-expandable ESV is likely to decrease annular eccentricity as it expands to circular shape in vivo $[3,12]$. Thus, the ESV size derived from the annular area and $\mathrm{D}_{\mathrm{A}}$ only agreed in $50 \%$ of cases. Also the perimeter and
Fig. 2 Use of one-dimensional diameters under the assumption of aortic annulus circularity may lead to underestimation (green circle; short diameter) or overestimation (red circle; long diameter) of optimal aortic valve prosthesis size. red line-short diameter; blue line-long diameter; green circular area-area of circle calculated from short diameter; red circular area-area of circle calculated from long diameter; light blue-outline/perimeter of native aortic annulus

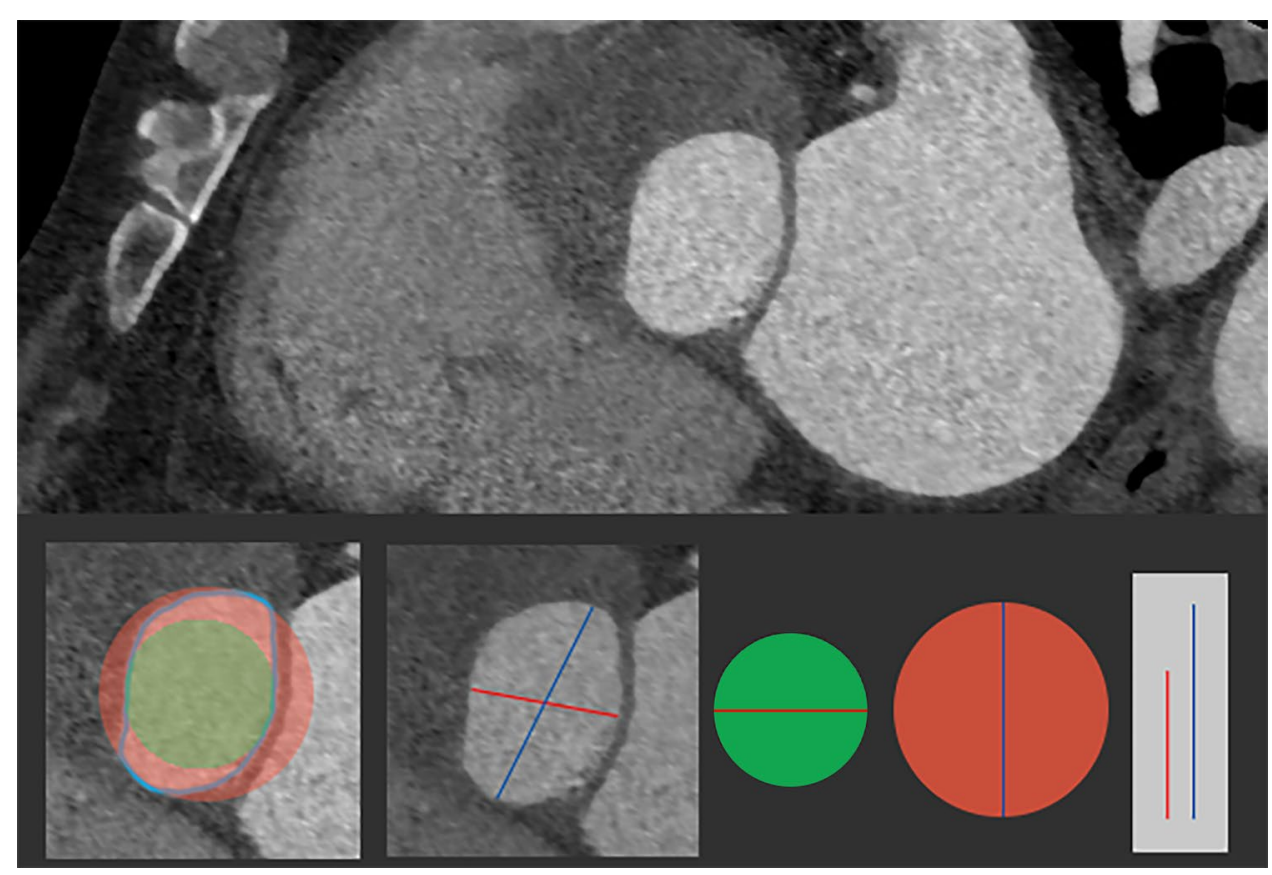


Fig. 3 The area of the circle and all ellipses presented in this figure is constant $\left(4.5 \mathrm{~cm}^{2}\right)$. Figure showing how measure of perimeter increases with eccentricity of the elliptical aortic annulus and respective increase in the prosthesis valve size. full blue figure-circle or ellipse with an area of $4.5 \mathrm{~cm}^{2}$; blue outline-perimeter of circle or ellipse with different eccentricity, while the area of $4.5 \mathrm{~cm}^{2}$ is kept constant; green labelESV valve size derived from the measurement (aortic annulus area, diameter or perimeter); red label-MCV valve size derived from the measurement (aortic annulus area, diameter or perimeter). $\mathrm{cm}^{2}$ square centimeter, $E S V$ Edwards Sapien XT Valve, $M C V$ Medtronic CoreValve, $\mathrm{mm}$ millimeter
A
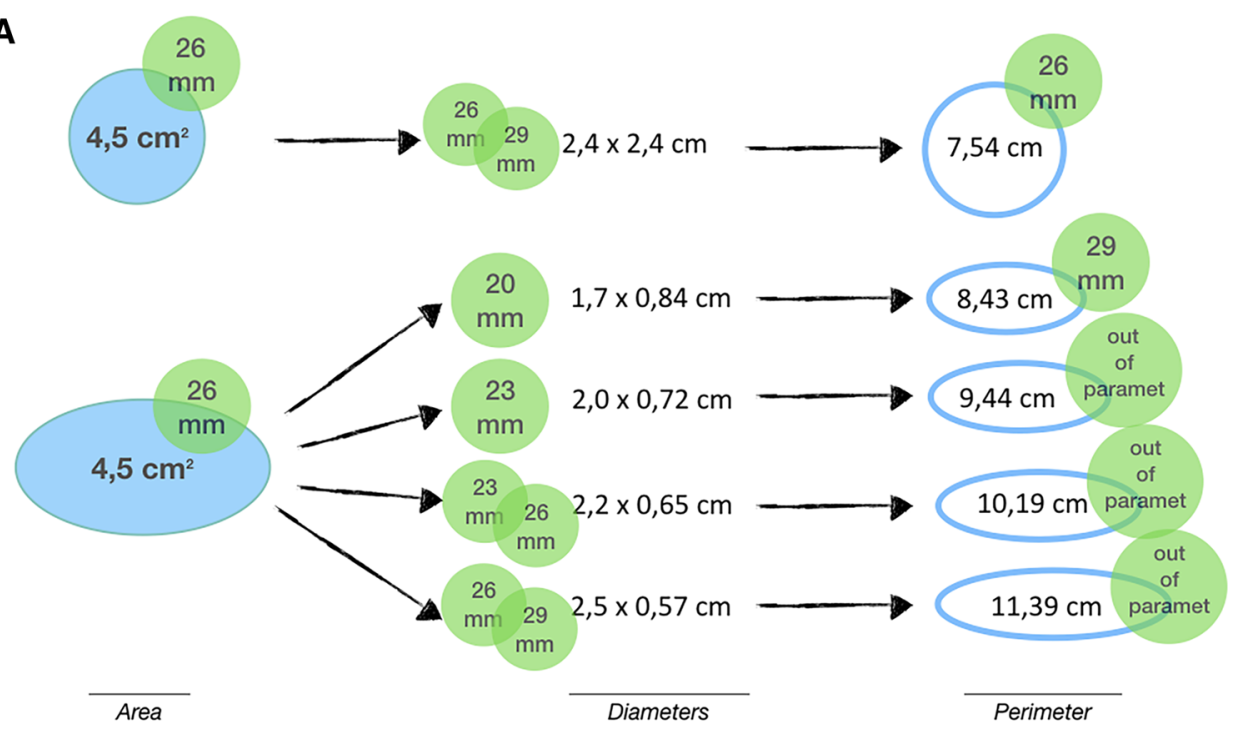

Edwards Sapien XT Valve

B
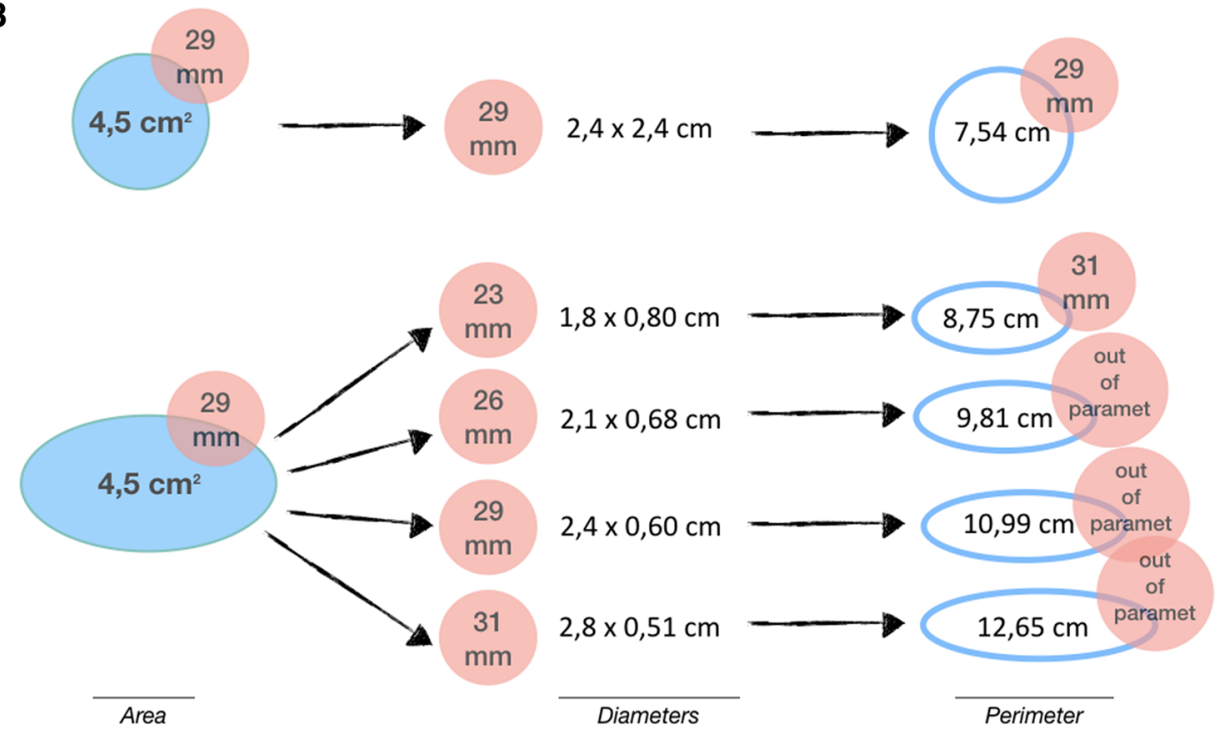

Medtronic Core Valve
$\mathrm{D}_{\mathrm{P}}$ would lead to selection of the same prosthesis size in only $48 \%$ and $84 \%$ of patients referred for ESV and MCV, respectively. It is not surprising, if the elliptical shape of the native aortic annulus is considered, that the use of $D_{P}$ consistently leads to selection of a larger valve compared to short diameter (76-82\%), $\mathrm{D}_{\mathrm{A}}(20-24 \%)$, area (24-50\%) and perimeter (16-54\%; Table 8 in Appendix 3). Kim et al. have confirmed this overestimation also in comparison to the direct surgical sizing [19]. Based on our results, we believe that effective diameters should not be preferred over direct annular measurements, because they represent an indirect calculation, which assume a perfect annular circularity and therefore are prone to computational errors.

This study has demonstrated that the use of area-derived sizing thresholds in TAVI candidates results in consensus in prosthesis size with at least one other annular dimension in all patients, regardless on the valve type. The measurement of annular area appears to correspond the best with the industry recommended sizing thresholds because the shape of the native aortic annulus does not affect the measure of its area [20]. Together with reports of high inter- and intrareader reproducibility [15], our results suggest that annular area is the most robust parameter in transcatheter aortic 
valve prosthesis size selection. Moreover, there might be additional benefits to the use of annular area in transcatheter aortic valve sizing. Willson et al. found the TAVI prosthesis sizing derived from the aortic annulus area to significantly reduce the risk of moderate or severe post-procedural paravalvular leakage (area under the curve: 0.80) [21]. Blanke et al. reported the annular area measurements on MDCT to be the best correlated parameter on pre- and post-TAVI datasets [12]. We therefore assume that eventual reshaping of aortic annulus after valve deployment is not likely to significantly influence annular area, therefore its consistency may allow for further and more sophisticated determination of necessary oversizing in order to minimize the risk of both paravalvular leak and annular injury.

\section{Limitations}

This study is subject to some limitations. First of all, a relatively small population was evaluated. Although this study used annular measurements of actual TAVI candidates and thus mimics the common clinical decision-making process in transcatheter aortic valve prosthesis sizing, the results were established theoretically and should be viewed as hypothesis generating. Additionally, we have only evaluated the manufacturer recommendations for ESV and MCV devices, thus our results may not extrapolate to prosthetic valves of other manufacturers. This study assumed that after in vivo deployment the prosthesis would expand to its nominal size reported by the manufacturer. The retrospective character of this study does not permit confrontation of our results with clinical outcomes, procedural success or patientprosthesis mismatch, because multiple transcatheter aortic devices were used during the evaluated period.

\section{Conclusion}

Aortic annulus area appears to be the most robust parameter in transcatheter aortic valve prosthesis size selection. Short aortic annulus diameter may have a tendency to lead to selection of one size smaller prosthesis size, while use of annular perimeter may lead to selection of one size larger prosthesis size.

Funding The authors state that this work has not received any funding.

\section{Compliance with ethical standards}

Conflict of interest The conflict of interest is none within the manuscript.

Ethical approval All procedures performed in studies involving human participants were in accordance with the ethical standards of the institutional and/or national research committee and with the 1964 Helsinki declaration and its later amendments or comparable ethical standards. The local medical ethical research committee reference number: 15-4076.

Open Access This article is distributed under the terms of the Creative Commons Attribution 4.0 International License (http://creat ivecommons.org/licenses/by/4.0/), which permits unrestricted use, distribution, and reproduction in any medium, provided you give appropriate credit to the original author(s) and the source, provide a link to the Creative Commons license, and indicate if changes were made.

\section{Appendix 1}

See Table 6.
Table 6 Mean annular dimensions derived from echocardiography (LVOT) and MDCT

\begin{tabular}{lllll}
\hline Diameter & Mean \pm SD $[\mathrm{mm}]$ & Mean difference from LVOT & $95 \%$ LOA & Sign. (2-sided) \\
\hline LVOT & $21.1 \pm 1.4$ & & & $\mathrm{p}<0.001$ \\
Short & $22.3 \pm 1.7$ & $1.3 \pm 1.8$ & -2.2 to 4.8 & $\mathrm{p}<0.001$ \\
$\mathrm{D}_{\mathrm{A}}$ & $24.5 \pm 1.7$ & $3.4 \pm 1.7$ & 0.1 to 6.7 & $\mathrm{p}<0.001$ \\
Mean & $25.0 \pm 1.7$ & $4.0 \pm 1.7$ & 0.7 to 7.3 & $\mathrm{p}<0.001$ \\
$\mathrm{D}_{\mathrm{P}}$ & $25.2 \pm 1.8$ & $4.2 \pm 1.8$ & 0.7 to 7.3 & $\mathrm{p}<0.001$ \\
Long & $27.7 \pm 2.0$ & $6.7 \pm 2.0$ & 2.8 to 10.6 & $\mathrm{p}<0.001$ \\
\hline
\end{tabular}

$D_{A}$ area derived effective diameter, $D_{p}$ perimeter derived effective diameter, $L O A$ limits of agreement, $L V O T$ left ventricular outflow tract, $M D C T$ multidetector computed tomography, $m m$ millimeter, $S D$ standard deviation, sign. significance 


\section{Appendix 2}

See Table 7 and Fig. 4.

Table 7 Transcatheter aortic valve size distribution derived from annular diameters (shown as number of cases)

\begin{tabular}{|c|c|c|c|c|c|c|c|c|c|}
\hline \multirow{2}{*}{$\begin{array}{l}\text { Valve } \\
\text { size/annular } \\
\text { diameter }\end{array}$} & \multicolumn{4}{|c|}{ ESV } & \multicolumn{5}{|c|}{$\mathrm{MCV}$} \\
\hline & $\begin{array}{l}23 \\
\mathrm{~mm}\end{array}$ & $\begin{array}{l}26 \\
\mathrm{~mm}\end{array}$ & $\begin{array}{l}29 \\
\mathrm{~mm}\end{array}$ & $\begin{array}{l}\text { Not } \\
\text { suitable }\end{array}$ & $\begin{array}{l}23 \\
\mathrm{~mm}\end{array}$ & $\begin{array}{l}26 \\
\mathrm{~mm}\end{array}$ & $\begin{array}{l}29 \\
\mathrm{~mm}\end{array}$ & $\begin{array}{l}31 \\
\mathrm{~mm}\end{array}$ & $\begin{array}{l}\text { Not } \\
\text { suitable }\end{array}$ \\
\hline Short & 16 & 25 & 9 & 0 & 4 & 28 & 18 & 0 & 0 \\
\hline $\mathrm{D}_{\mathrm{A}}$ & 3 & 16 & 31 & 0 & 0 & 9 & 38 & 3 & 0 \\
\hline Mean & 2 & 12 & 36 & 0 & 0 & 8 & 37 & 5 & 0 \\
\hline $\mathrm{D}_{\mathrm{P}}$ & 2 & 11 & 34 & 3 & 0 & 4 & 36 & 9 & 1 \\
\hline Long & 0 & 2 & 21 & 27 & 0 & 0 & 17 & 19 & 14 \\
\hline
\end{tabular}

$D_{A}$ area derived effective diameter, $D_{P}$ perimeter derived effective diameter, ESV Edwards Sapien XT Valve, $M C V$ Medtronic CoreValve, $m m$ millimeter, not suitable currently suitable valve size [ESV > $29 \mathrm{~mm}, \mathrm{MCV}>31 \mathrm{~mm}$ ] not available 


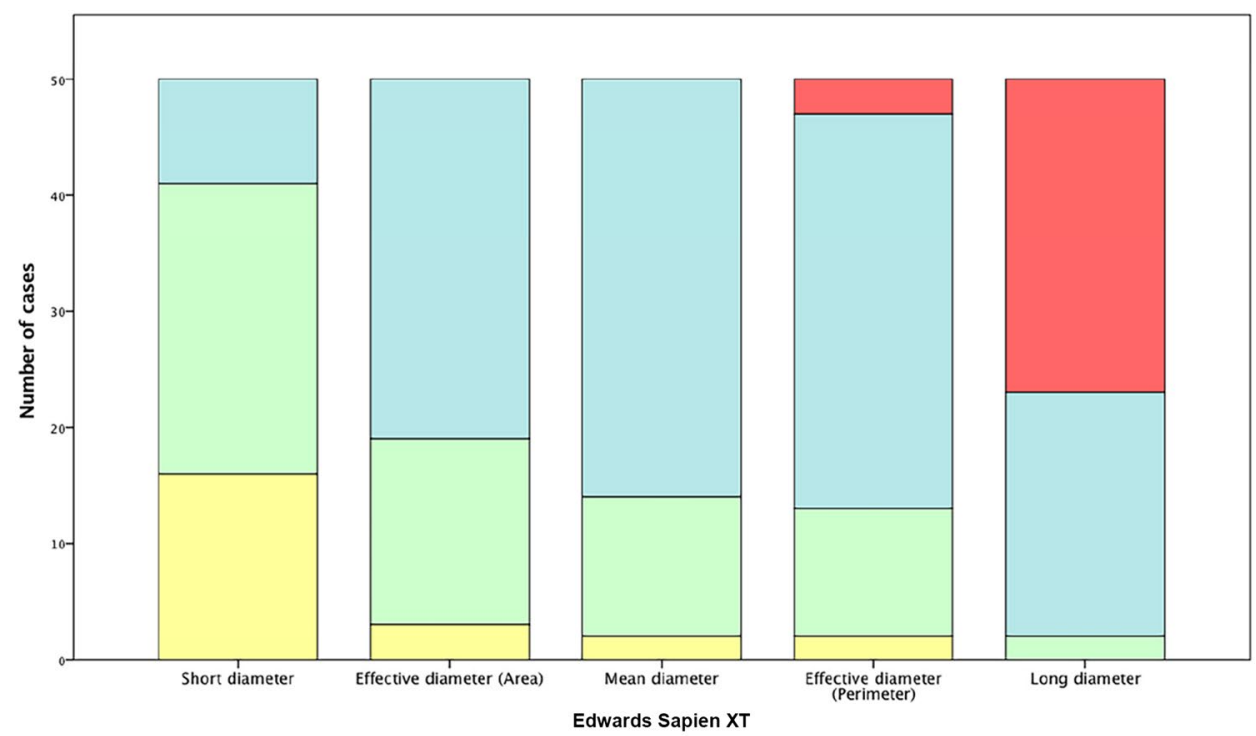

Valve Size

$\square$ Out of parameter

$29 \mathrm{~mm}$

$26 \mathrm{~mm}$

20.00

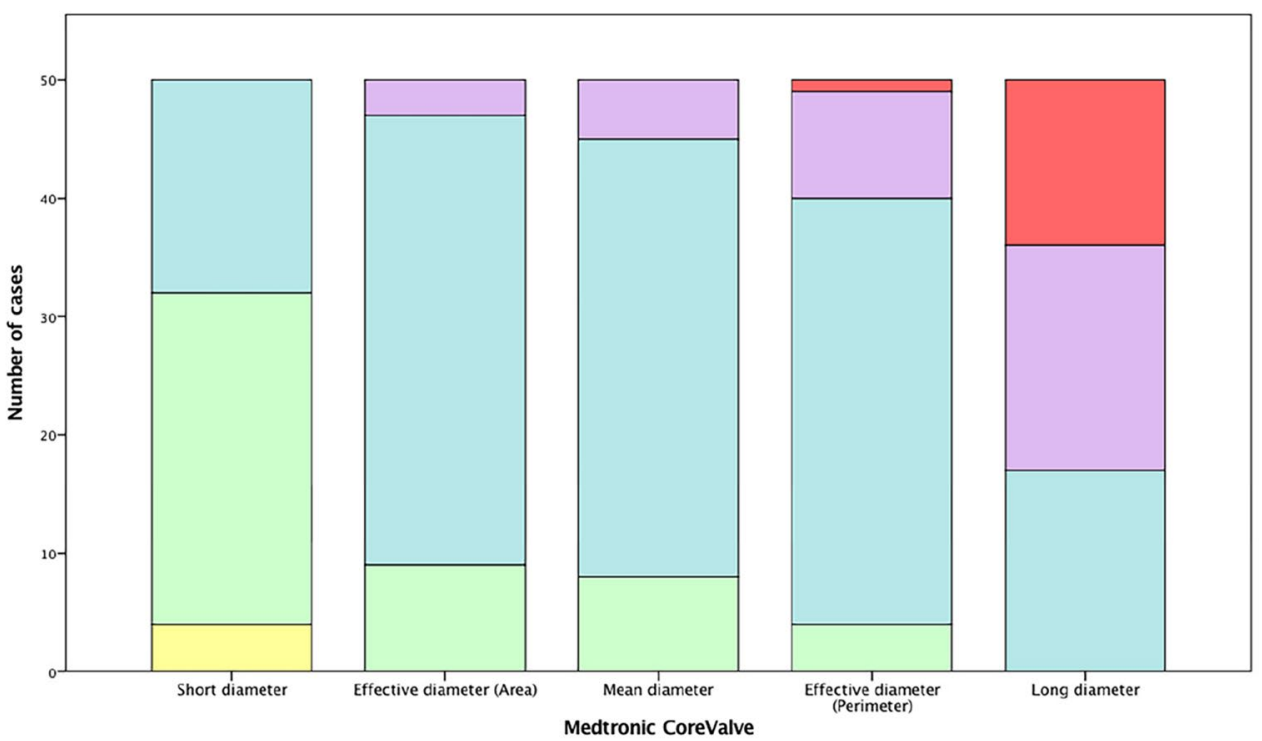

Valve Size

$\square$ Out of parameter

$\square 31 \mathrm{~mm}$

$29 \mathrm{~mm}$

$\square 26 \mathrm{~mm}$

Fig. 4 Transcatheter aortic valve size distribution derived from annular diameters (shown as number of cases). $D_{A}$ area derived effective diameter, $D_{P}$ perimeter derived effective diameter, $E S V$ Edwards

\section{Appendix 3}

See Table 8.
Sapien XT Valve, $M C V$ Medtronic CoreValve, $\mathrm{mm}$ millimeter, not suitable - currently suitable valve size $[\mathrm{ESV}>29 \mathrm{~mm}, \mathrm{MCV}>31$ $\mathrm{mm}$ ] not available

Table 8 Comparison of transcatheter aortic valve sizes derived from annular effective diameters, area and perimeter (number and \% of cases)

\begin{tabular}{lcc|cl}
\hline \multicolumn{3}{c}{ ESV } & \multicolumn{2}{c}{ MCV } \\
\hline \multicolumn{2}{l}{ Prosthesis size derived from } & & & \\
- Area $=\mathrm{D}_{\mathrm{A}}$ & 25 & $50 \%$ & 50 & $100 \%$ \\
- Perimeter $=\mathrm{D}_{\mathrm{P}}$ & 24 & $48 \%$ & 42 & $84 \%$ \\
- $\mathrm{D}_{\mathrm{P}}>\mathrm{D}_{\mathrm{A}}$ & 10 & $20 \%$ & 12 & $24 \%$ \\
- $\mathrm{D}_{\mathrm{P}}>$ area & 25 & $50 \%$ & 12 & $24 \%$ \\
- $\mathrm{D}_{\mathrm{P}}>$ perimeter & 27 & $54 \%$ & 8 & $16 \%$ \\
\hline
\end{tabular}

$D_{A}$ effective diameter derived from annular area, $D_{P}$ effective diameter derived from annular perimeter, $E S V$ Edwards Sapien Valve, $M C V$ Medtronic CoreValve 


\section{References}

1. Schwarz F, Lange P, Zinsser D, Greif M, Boekstegers P, Schmitz C, Reiser MF, Kupatt C, Becker HC (2014) CT-angiographybased evaluation of the aortic annulus for prosthesis sizing in transcatheter aortic valve implantation (TAVI)-predictive value and optimal thresholds for major anatomic parameters. PLoS ONE 9(8):e103481. https://doi.org/10.1371/journal.pone.0103481

2. Schmidkonz C, Marwan M, Klinghammer L, Mitschke M, Schuhbaeck A, Arnold M, Lell M, Achenbach S, Pflederer T (2014) Interobserver variability of CT angiography for evaluation of aortic annulus dimensions prior to transcatheter aortic valve implantation (TAVI). Eur J Radiol 83(9):1672-1678

3. Kasel AM, Cassese S, Bleiziffer S, Amaki M, Hahn RT, Kastrati A, Sengupta PP (2013) Standardized imaging for aortic annular sizing: implications for transcatheter valve selection. JACC Cardiovasc Imaging 6(2):249-262

4. Baumgartner H, Falk V, Bax JJ, De Bonis M, Hamm C, Holm PJ, Iung B, Lancellotti P, Lansac E, Rodriguez Munoz D, Rosenhek R, Sjogren J, Tornos Mas P, Vahanian A, Walther T, Wendler O, Windecker S, Zamorano JL, ESC Scientific Document Group (2017) 2017 ESC/EACTS guidelines for the management of valvular heart disease. Eur Heart J 38(36):2739-2791. https://doi. org/10.1093/eurheartj/ehx391

5. Jilaihawi H, Kashif M, Fontana G, Furugen A, Shiota T, Friede G, Makhija R, Doctor N, Leon MB, Makkar RR (2012) Crosssectional computed tomographic assessment improves accuracy of aortic annular sizing for transcatheter aortic valve replacement and reduces the incidence of paravalvular aortic regurgitation. J Am Coll Cardiol 59(14):1275-1286. https://doi.org/10.1016/j. jacc.2011.11.045

6. Achenbach S, Delgado V, Hausleiter J, Schoenhagen P, Min JK, Leipsic JA (2012) SCCT expert consensus document on computed tomography imaging before transcatheter aortic valve implantation (TAVI)/transcatheter aortic valve replacement (TAVR). J Cardiovasc Comput Tomogr 6(6):366-380

7. Smid M, Ferda J, Baxa J, Cech J, Hajek T, Kreuzberg B, Rokyta $\mathrm{R}$ (2010) Aortic annulus and ascending aorta: comparison of preoperative and periooperative measurement in patients with aortic stenosis. Eur J Radiol 74(1):152-155. https://doi.org/10.1016/j. ejrad.2009.01.028

8. Jurencak T, Turek J, Kietselaer BL, Mihl C, Kok M, van Ommen VG, van Garsse LA, Nijssen EC, Wildberger JE, Das M (2015) MDCT evaluation of aortic root and aortic valve prior to TAVI. What is the optimal imaging time point in the cardiac cycle? Eur Radiol 25(7):1975-1983. https://doi.org/10.1007/s0033 0-015-3607-5

9. Mylotte D, Martucci G, Piazza N (2012) Patient selection for transcatheter aortic valve implantation: an interventional cardiology perspective. Ann Cardiothorac Surg 1(2):206-215

10. Blanke P, Willson AB, Webb JG, Achenbach S, Piazza N, Min JK, Pache G, Leipsic J (2014) Oversizing in transcatheter aortic valve replacement, a commonly used term but a poorly understood one: dependency on definition and geometrical measurements. J Cardiovasc Comput Tomogr 8(1):67-76

11. Kempfert J, Van Linden A, Lehmkuhl L, Rastan AJ, Holzhey D, Blumenstein J, Mohr FW, Walther T (2012) Aortic annulus sizing: echocardiographic versus computed tomography derived measurements in comparison with direct surgical sizing. Eur J Cardiothorac Surg 42(4):627-633. https://doi.org/10.1093/ejcts /ezs064

12. Blanke P, Russe M, Leipsic J, Reinohl J, Ebersberger U, Suranyi P, Siepe M, Pache G, Langer M, Schoepf UJ (2012) Conformational pulsatile changes of the aortic annulus: impact on prosthesis sizing by computed tomography for transcatheter aortic valve replacement. JACC Cardiovasc Interv 5(9):984-994. https://doi. org/10.1016/j.jcin.2012.05.014

13. Schultz CJ, Moelker A, Piazza N, Tzikas A, Otten A, Nuis RJ, Neefjes LA, van Geuns RJ, de Feyter P, Krestin G, Serruys PW, de Jaegere PP (2010) Three dimensional evaluation of the aortic annulus using multislice computer tomography: are manufacturer's guidelines for sizing for percutaneous aortic valve replacement helpful? Eur Heart J 31(7):849-856. https://doi.org/10.1093/ eurheartj/ehp534

14. Popma JJ, Gleason TG, Yakubov SJ, Harrison JK, Forrest JK, Maini B, Ruiz CE, Pinto DS, Costa M, Resar J, Conte J, Crestanello J, Chang Y, Oh JK, Reardon MJ, Adams DH (2016) Relationship of annular sizing using multidetector computed tomographic imaging and clinical outcomes after self-expanding corevalve transcatheter aortic valve replacement. Circ Cardiovasc Interv 9 (7). https://doi. org/10.1161/CIRCINTERVENTIONS.115.003282

15. Willson AB, Webb JG, Freeman M, Wood DA, Gurvitch R, Thompson CR, Moss RR, Toggweiler S, Binder RK, Munt B, Cheung A, Hague C, Ye J, Leipsic JA (2012) Computed tomography-based sizing recommendations for transcatheter aortic valve replacement with balloon-expandable valves: comparison with transesophageal echocardiography and rationale for implementation in a prospective trial. J Cardiovasc Comput Tomogr 6(6):406-414

16. Abdel-Wahab M, Zahn R, Horack M, Gerckens U, Schuler G, Sievert H, Eggebrecht H, Senges J, Richardt G, Gerckens U, Schuler G, Zahn R, Eggebrecht H, Sievert H, Hauptmann KE, Kuck KH, Hambrecht R, Richardt G, Nickenig G, Naber CH, Sack S, Figulla HR, Block M, Hoffmann E, Sechtem U, Gulker H, Riegger G, Mudra H, Neumann FJ, Bode C, Brachmann J (2011) Aortic regurgitation after transcatheter aortic valve implantation: incidence and early outcome. Results from the German transcatheter aortic valve interventions registry. Heart 97(11):899-906

17. Athappan G, Patvardhan E, Tuzcu EM, Svensson LG, Lemos PA, Fraccaro C, Tarantini G, Sinning J-M, Nickenig G, Capodanno D, Tamburino C, Latib A, Colombo A, Kapadia SR (2013) Incidence, predictors, and outcomes of aortic regurgitation after transcatheter aortic valve replacement: meta-analysis and systematic review of literature. J Am Coll Cardiol 61(15):1585-1595

18. von Aspern K, Foldyna B, Etz CD, Hoyer A, Girrbach F, Holzhey D, Lucke C, Grothoff M, Linke A, Mohr FW, Gutberlet M, Lehmkuhl L (2015) Effective diameter of the aortic annulus prior to transcatheter aortic valve implantation: influence of area-based versus perimeterbased calculation. Int J Cardiovasc Imaging 31(1):163-169

19. Kim W-K, Meyer A, Mollmann H, Rolf A, Mollmann S, Blumenstein J, Van Linden A, Hamm CW, Walther T, Kempfert J (2016) Cyclic changes in area- and perimeter-derived effective dimensions of the aortic annulus measured with multislice computed tomography and comparison with metric intraoperative sizing. Clin Res Cardiol 105(7):622-629

20. Lehmkuhl L, Foldyna B, Von Aspern K, Lucke C, Grothoff M, Nitzsche S, Kempfert J, Haensig M, Rastan A, Walther T, Mohr F-W, Gutberlet M (2013) Inter-individual variance and cardiac cycle dependency of aortic root dimensions and shape as assessed by ECG-gated multi-slice computed tomography in patients with severe aortic stenosis prior to transcatheter aortic valve implantation: is it crucial for correct sizing? Int J Cardiovasc Imaging 29(3):693-703

21. Willson AB, Webb JG, Labounty TM, Achenbach S, Moss R, Wheeler M, Thompson C, Min JK, Gurvitch R, Norgaard BL, Hague CJ, Toggweiler S, Binder R, Freeman M, Poulter R, Poulsen S, Wood DA, Leipsic J (2012) 3-dimensional aortic annular assessment by multidetector computed tomography predicts moderate or severe paravalvular regurgitation after transcatheter aortic valve replacement: a multicenter retrospective analysis. J Am Coll Cardiol 59(14):1287-1294. https://doi.org/10.1016/j. jacc.2011.12.015 